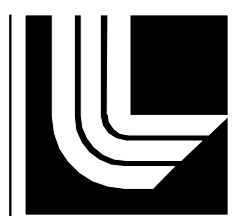

Aftershock Analysis for SPE2

LAWRENCE LIVERMORE NATIONAL LABORATORY

Jerry Sweeney and Phil Harben

October 22, 2012 
This work performed under the auspices of the U.S. Department of Energy by Lawrence Livermore National Laboratory under Contract DE-AC52-07NA27344.

\section{Disclaimer}

This document was prepared as an account of work sponsored by an agency of the United States government. Neither the United States government nor Lawrence Livermore National Security, LLC, nor any of their employees makes any warranty, expressed or implied, or assumes any legal liability or responsibility for the accuracy, completeness, or usefulness of any information, apparatus, product, or process disclosed, or represents that its use would not infringe privately owned rights. Reference herein to any specific commercial product, process, or service by trade name, trademark, manufacturer, or otherwise does not necessarily constitute or imply its endorsement, recommendation, or favoring by the United States government or Lawrence Livermore National Security, LLC. The views and opinions of authors expressed herein do not necessarily state or reflect those of the United States government or Lawrence Livermore National Security, LLC, and shall not be used for advertising or product endorsement purposes. 


\title{
Aftershock Analysis for SPE2
}

\author{
Jerry Sweeney and Phil Harben \\ Lawrence Livermore National Laboratory
}

\section{Introduction}

This is the second report on a study of possible seismic aftershocks associated with chemical explosions in granitic rock. The Source Physics 2 experiment (SPE2), consisting of a $2000 \mathrm{~kg}$ TNT equivalent chemical explosion at a depth of $45 \mathrm{~m}$, was detonated on October 25, 2011 (day 298). A report by Sweeney and Harben (2011) describes results of aftershock analysis of the SPE1 experiment - a smaller $100 \mathrm{~kg}$ explosion at $60 \mathrm{~m}$ depth -- that took place in the same location on May 3, 2011.

Aftershock detection and location is an important element of an on-site inspection (OSI) under the Comprehensive Nuclear Test Ban Treaty (CTBT). Aftershocks have been observed to occur in the immediate vicinity of the detonation following nuclear explosions and thus local aftershock monitoring has been included in the CTBT as a method to locate the site of a clandestine underground nuclear test during an OSI. Thus the seismic aftershock monitoring system (SAMS) is an important element of the search procedure during an OSI. Because the frequency of occurrence and magnitude of the aftershocks decay with time following the explosion (Adushkin and Spivak, 1995; Ryall and Savage, 1969), it is important that the inspection be carried out as soon as possible after detection of a suspect event, and this aspect of inspections has been considered in the CTBT Protocol. Because of limited experience with aftershock monitoring, there is large uncertainty about the details of the aftershock process: effects of geology on aftershock rates; effects of initial explosion magnitude on aftershock magnitude; and effects of the seismic source on the distribution of aftershocks in the vicinity of the explosion. Some of these issues have been addressed in recent work (Ford and Walter, 2010; Ford et al, 2011).

One issue noted by Sweeney and Harben (2011) is the possibility that a zone of aftershock activity can be detected via elevated seismic noise in the region after the level of aftershocks has decayed to the point where individual events cannot be identified. If this approach - looking for elevated seismic noise in a particular frequency band - works, then the time for application of SAMS in an OSI could be extended. Thus, in addition to quantifying aftershock decay rates, we are examining the spectral and spatial character of possible elevated noise in the vicinity of the explosion following detonation.

Results of the SPE1 explosion were surprising because no aftershocks were observed. In the report from that experiment, we noted problems with coupling and site effects on the geophones that could elevate noise, but in spite of these issues, theory based on larger explosions suggested that we should have seen some very low magnitude aftershocks from this explosion. A possible contributing factor in this 
result is that the explosion was small and buried relatively deeply. The damage effects from this explosion were small and there was no explosion cavity - thus no free face of rock for movement underground that would cause aftershocks. Our hope was that the much larger SPE2 explosion, which was detonated at a shallower depth, would have a much larger potential to create aftershocks.

\section{Experiment layout, data collection, and results}

The same configuration of instruments was used for both SPE1 and SPE2. For SPE2 an effort was made to improve the coupling of the geophones and rewire connections to minimize noise and cross coupling between instruments. As was done for SPE1, we analyzed one week of continuous data immediately following the explosion for the presence of aftershocks. For SPE2 we visually examined waveforms from the three closest stations (located 100, 200, and 300 meters from ground zero) on each of the five radial lines of geophones as shown below (Fig. 1). The geophones used are model Geosys GS11D (see Mellors et al., 2012) for details about settings and performance of the instruments. Time coincident arrivals on the three lines at the same ranges with a consistent time delay from the $100 \mathrm{~m}$ ring to the $200 \mathrm{~m}$ and $300 \mathrm{~m}$ ring would be highly indicative of an aftershock near the working point. We did not observe any patterns fitting these criteria and we conclude that, as observed for SPE1, the SPE2 did not produce detectable aftershocks during the recording times available.

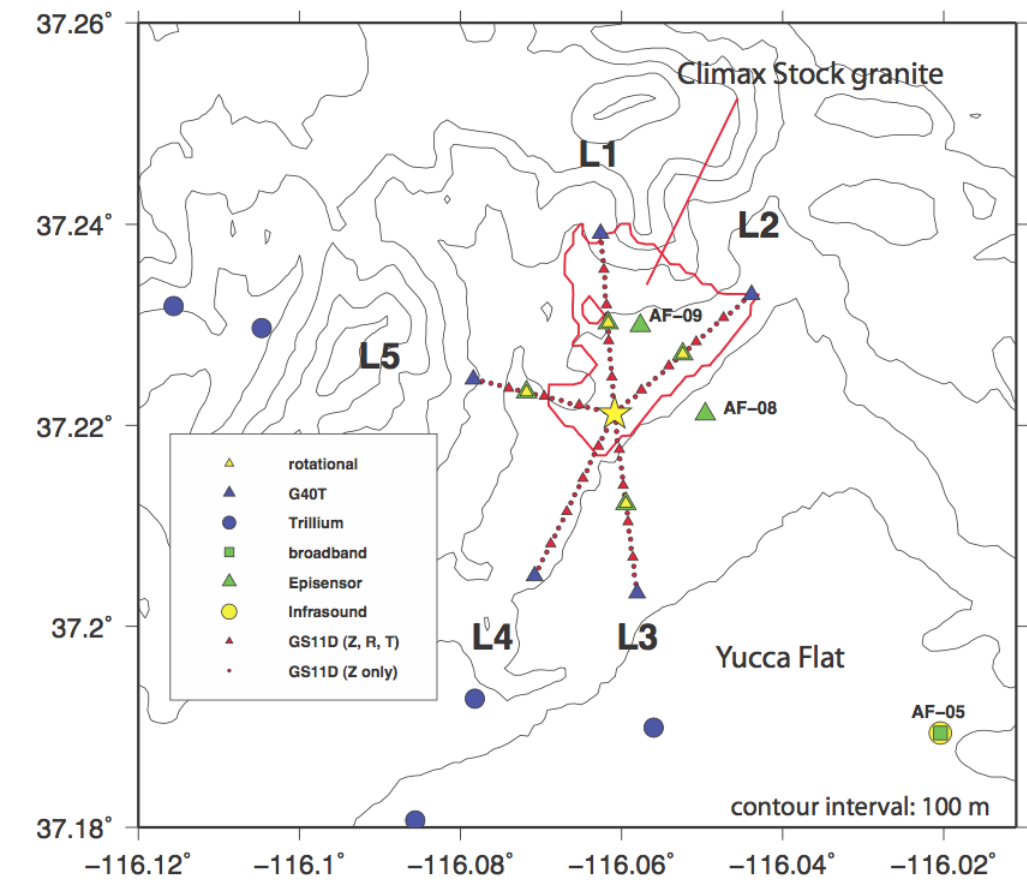

Figure 1. Map showing the layout of seismic instrumentation for the two explosion experiments. There are lines of instruments radiating out from the center point, which is the hypocenter (ground zero) of SPE1 and SPE2. Aftershock recordings were analyzed for the three closest stations to ground zero on five lines. 


\section{Aftershock Detection Capability of the SPE Temporary Seismic Network}

To assess aftershock detectability for the network we used the vertical geophones located from 100 to $1000 \mathrm{~m}$ from ground zero. We determined the signal-to-noise ratio (SNR) from the explosion by measuring the initial P-wave amplitude and dividing it by the root-mean-square (RMS) of the seismic background noise during a stationary noise period shortly before or after the explosion. The minimum detectability of an event is taken (after Rodgers, 1992) as an event with an SNR of 3 $\mathrm{dB}$, or 1.41. An excel spreadsheet for each of the two shots is the product of the SNT analysis; it contains the easting and northing coordinates of the geophones in meters and a third column with the base $10 \log$ of the SNR.

\section{SPE1 and SPE2 SNR}

The log of the SNR for SPE1 is shown in Fig. 2 for comparison with a similar plot for SPE2 (Fig. 3). Note that the locations of the stations used are shown with open circles. A station was not used if the signal or noise was an order of magnitude or more different than the trend would suggest. Plots consist of maps of the locations of stations to distances of 1000 meters from ground zero and the color intensity is the $\mathrm{SN}$, which ranges from about 2.5 to 5.0. The horizontal and vertical scales are arbitrary units of length; the spacing between stations on the same line is about 100 meters. The two plots of Figs. 2 and 3 are quite similar; Fig. 3 suggests that the SN dropped off slightly faster away from GZ for SPE2. However, in these studies we are primarily concerned with the three stations on each line closest to GZ and the plots indicate that the ability to detect aftershocks for each explosion is about the same. 


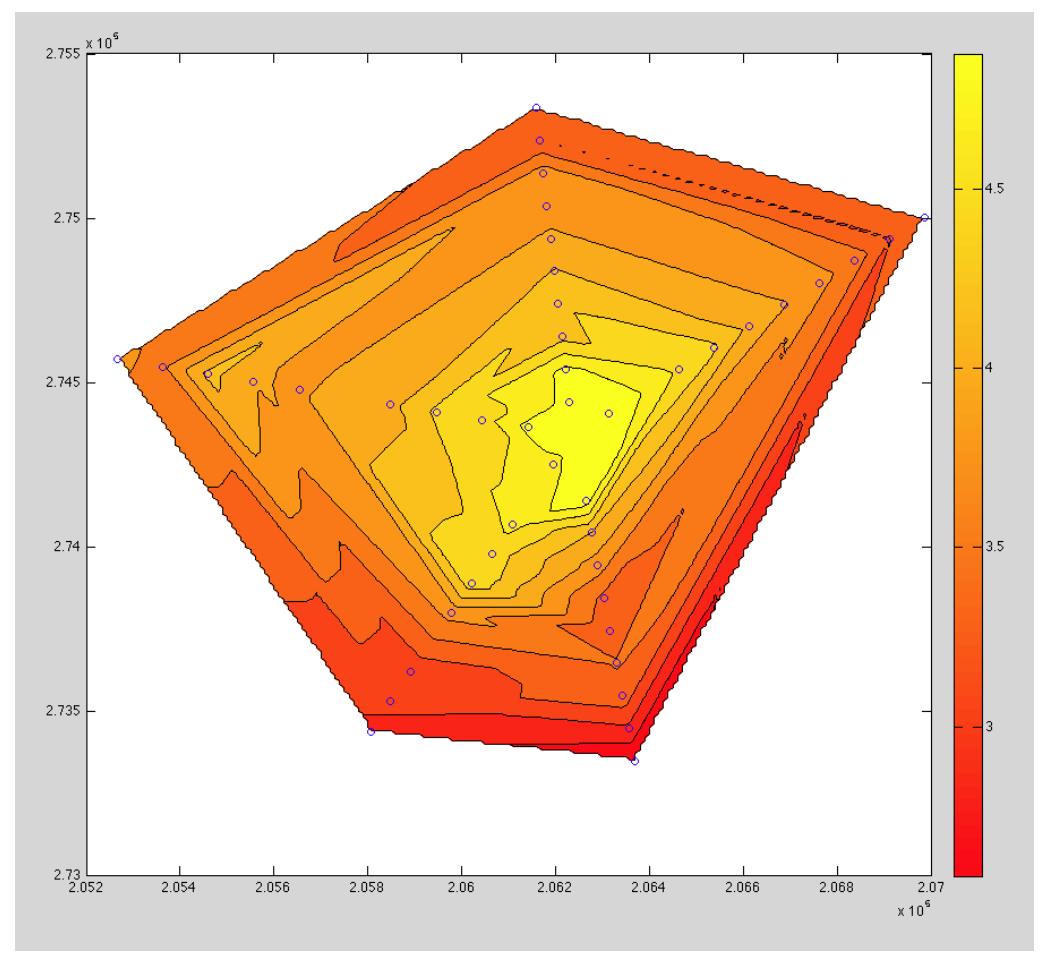

Figure 2. Signal to noise ratio versus station location for SPE1. Circles are station locations, with distances between stations along the lines about $100 \mathrm{~m}$.

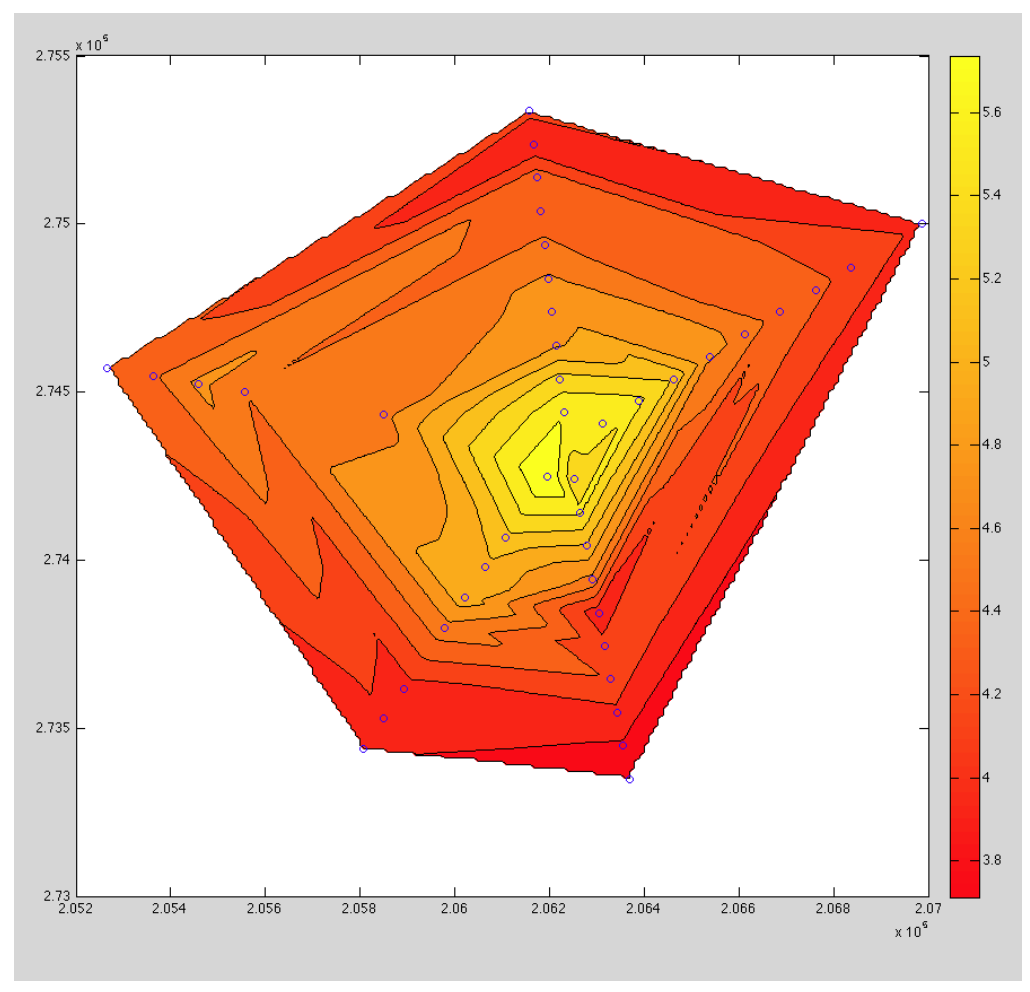

Figure 3. Signal to noise ratio versus station location for SPE2. Circles are station locations, with distances between stations along the lines about $100 \mathrm{~m}$. 


\section{SPE1 and SPE2 Determined Aftershock Detectability}

The detectability the network provides for aftershocks that occur in the immediate vicinity of the SPE borehole (within about 100 meters) is determined below using both the SPE1 and SPE2 SNs. The method uses the magnitude of the main SPE explosion and subtracts the $\mathrm{SN}$ in log space at each station. The result is the minimum magnitude detectable at each station. This assumes a magnitude-yield slope of 1.0, which may not be the case. However, since we do not have better information, it will have to suffice for these estimates. It should be noted that the magnitude detectability at the perimeter of the colored region can roughly set a conservative detection level for an aftershock that occurs anywhere in the colored region.

By doing a least squares fit to minimize residuals, the SPE1 and SPE2 detectability results indicate the magnitude difference between the two shots was 0.69 . The "official" local magnitudes for the events as determined by UNR using the Nevada Seismic Network are 0.27-0.42 for SPE1 and 1.25 for SPE2. Using these magnitudes, and taking the 0.42 value for SPE1 since it is closest to giving a magnitude difference consistent with the least squares fit of the $\mathrm{S} / \mathrm{N}$ data, we calculate new contour plots for SPE1 and SPE2 that reflect local detectability in magnitude. The two contour plots (shown in Figs. 4 and 5) are presented using the same contour levels so a direct visual comparison is possible. The plots are similar to those of Figs. 2 and 3 in that the map view shows locations of the stations. The color scale in this case is the computed minimum magnitude calculation for a given location. 


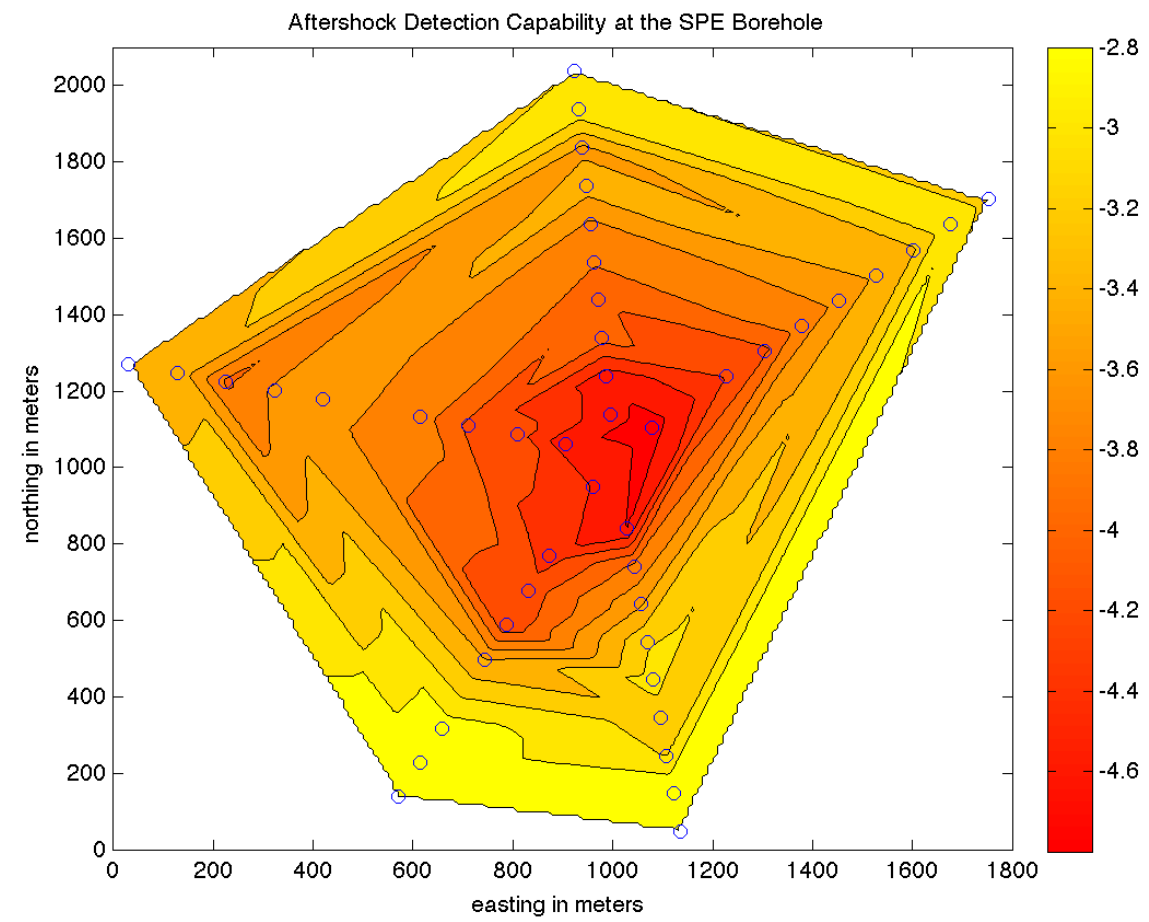

Figure 4. Minimum detection magnitude for SPE1 using the same SNR network of seismic sensors as shown in Figs. 2 and 3. Station separation along the lines is about $100 \mathrm{~m}$.

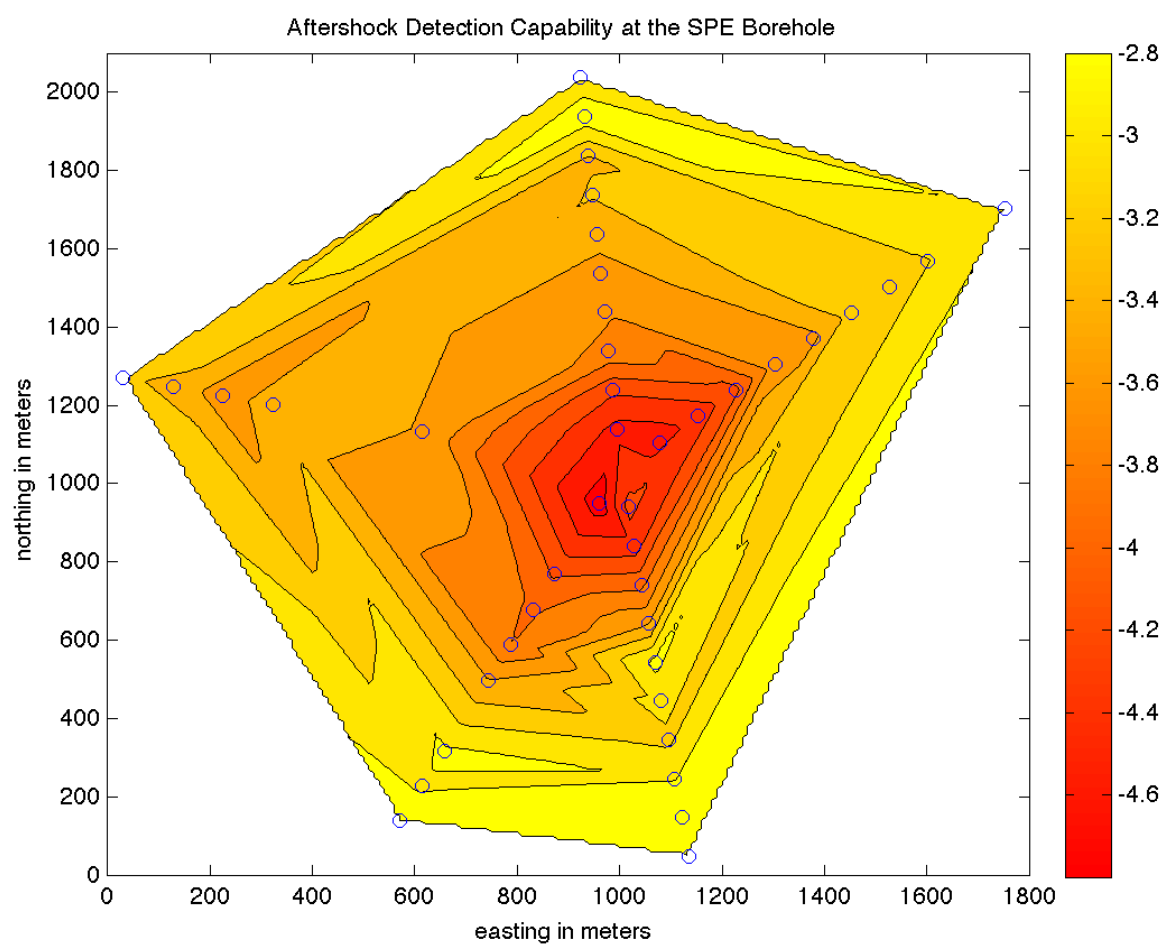

Figure 5. Minimum detection magnitude for SPE2 using the same SNR and network of seismic sensors as shown in Figs. 2 and 3. Station separation along the lines is about $100 \mathrm{~m}$. 


\section{SPE1 and SPE2 Aftershock Determination}

Continuous recording after the SPE1 explosion provided 10 days of post-event continuous data. A search for aftershocks was performed on this data but none were found. The results can be found in Harben and Sweeney (2011).

For SPE2, 10 days of continuous post-event data was recorded (days 298-308). The data was visually inspected over the entire period using the innermost stations $(100 \mathrm{~m}, 200 \mathrm{~m}$, and $300 \mathrm{~m})$. The procedure we used was to look at each one-hour record at station along a line going outward from GZ (e.g., L1-1, L1-2, and L1-3). If a short transient event was identified, the zoomed record was inspected for move out (e.g progressive delay in arrival due to the location of the station), with the greater ranges having later arrival times. If move out is observed and consistent with an event at the SPE2 source then the stations at the same distance from GZ (e.g., L1-1, L2-1, L3-1, L4-1, and L5-1) were inspected for the transient event arrival time. If these arrival times are nearly identical, then the event becomes a suspected aftershock.

Although many transient events were observed, none met the above criteria to become a suspected aftershock. The estimated minimum SNR among the transient events we studied was 10 or greater. Using the aftershock detection capability contour plot for SPE2, this corresponds to an aftershock detectability of about magnitude -3.0 at the inner stations. Consequently, we conclude that there were no aftershocks observed from the SPE2 event above magnitude -3 during the first 10 days after the event.

\section{Influence of Background Noise}

The background noise was evaluated during "quiet time" at NTS from day 299 to day 308 on the station $100 \mathrm{~m}$ from GZ on line 1, vertical component. Quiet time is when cultural noise can usually be expected to be minimal: midnight until $6 \mathrm{AM}$. High seismic noise due to weather and unusual events can, of course, occur at any time. We calculated the power density spectra for each one-hour waveform recording from midnight until 6 AM for days 299 to 308. Figs. 6 - 11 below show the spectra for each hour during the quiet time for the day labeled. The $y$-axis is in digitizer counts with a gain of one. This corresponds to a least significant bit value of $1.589 \times 10^{-6} \mathrm{~V} /$ count. The lowest broadband noise levels seen in the plots have maximum count levels of only 10 . For the GS11D geophone, with a generator constant of $0.32 \mathrm{~V} / \mathrm{cm} / \mathrm{s}$, this corresponds to a noise level of about $5 \times 10^{-7} \mathrm{~m} / \mathrm{s}$. Of particular note is the flatness and consistent floor of the spectra. This is probably due to the bit noise floor set by the least significant bit of the Reftek digitizer. Increasing the gain of the recording system would probably result in more bit resolution at such low noise periods and provide more noise structure. It cannot be ruled out, however, that these low noise levels are approaching the noise floor of the 
geophone. Increasing the gain would probably alter the noise floor and would also increase the risk of clipping should an SPE aftershock occur. This is because the current generation of deployed Reftek digitizers offer only two gain settings: $1 \mathrm{x}$ and $32 \mathrm{x}$.

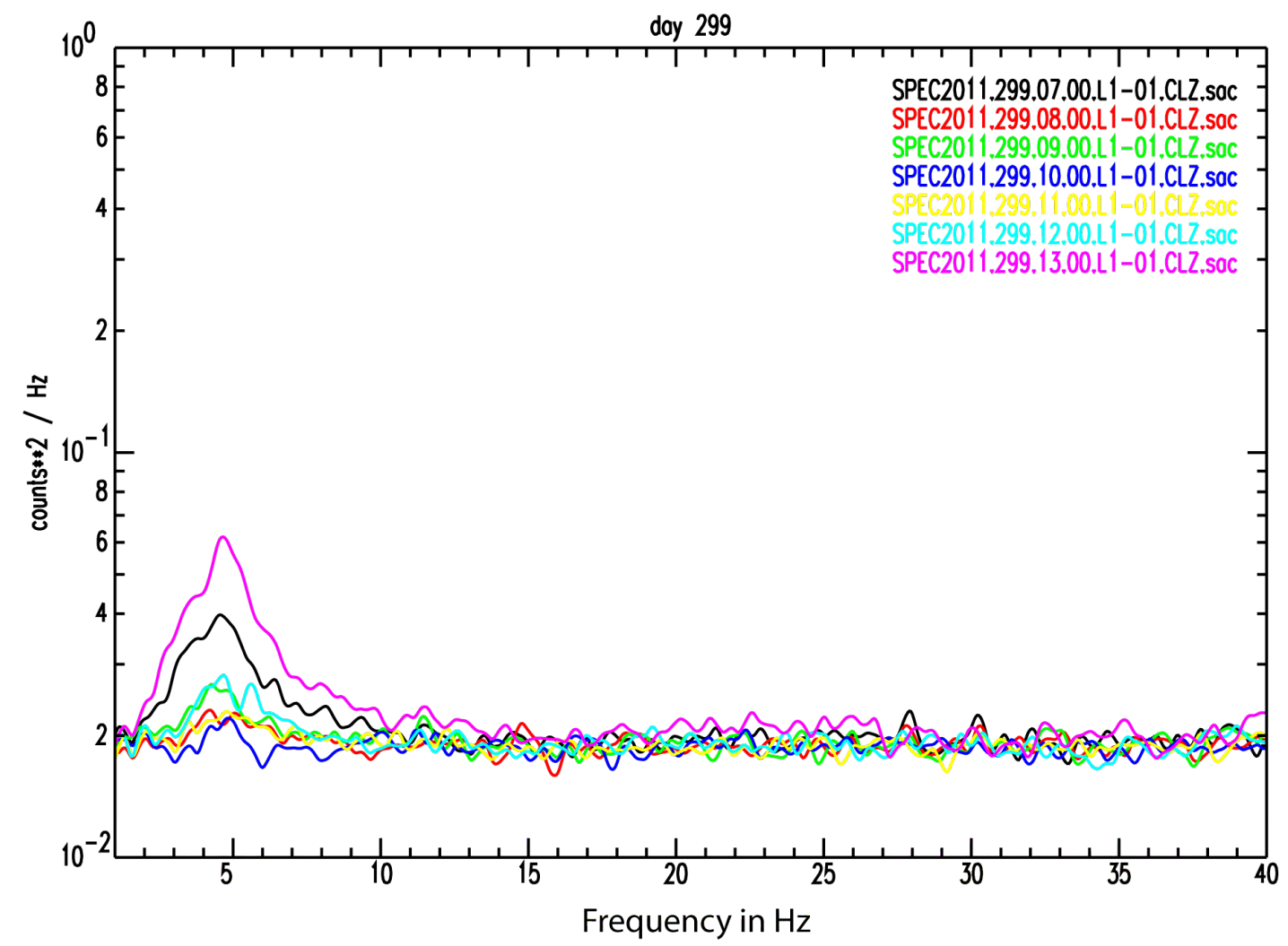

Figure 6. Power spectral density for each of seven consecutive hours after midnight local time $(0700$ through 1300 UT) on day 299 (evening after SPE2 was detonated) for the station $100 \mathrm{~m}$ from GZ along line 1 (refer to Fig 1). 


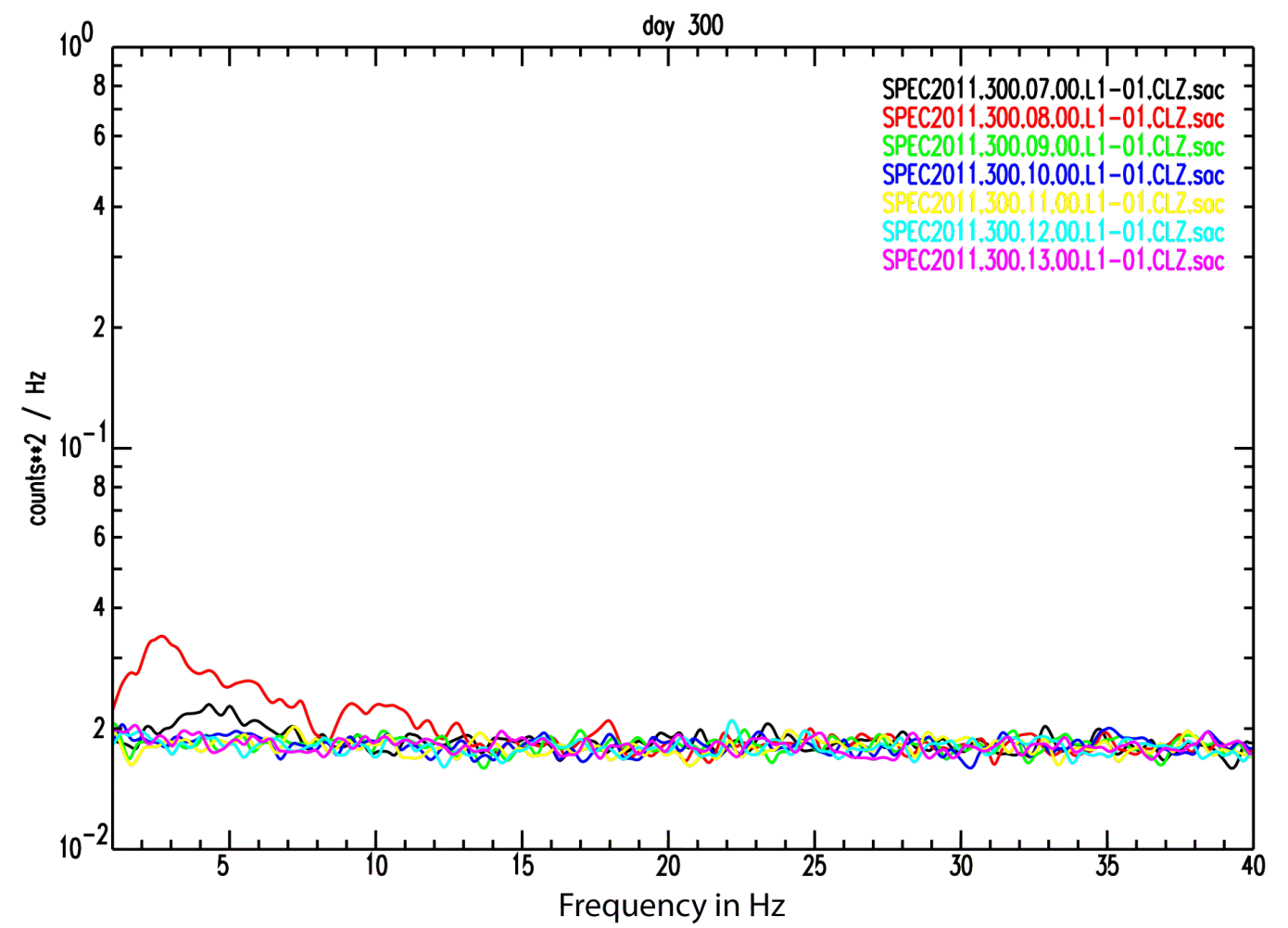

Figure 7. Power spectral density for each of seven consecutive hours after midnight local time (0700 through 1300 UT) on day 300 for the station 100 m from GZ along line 1 (refer to Fig 1).

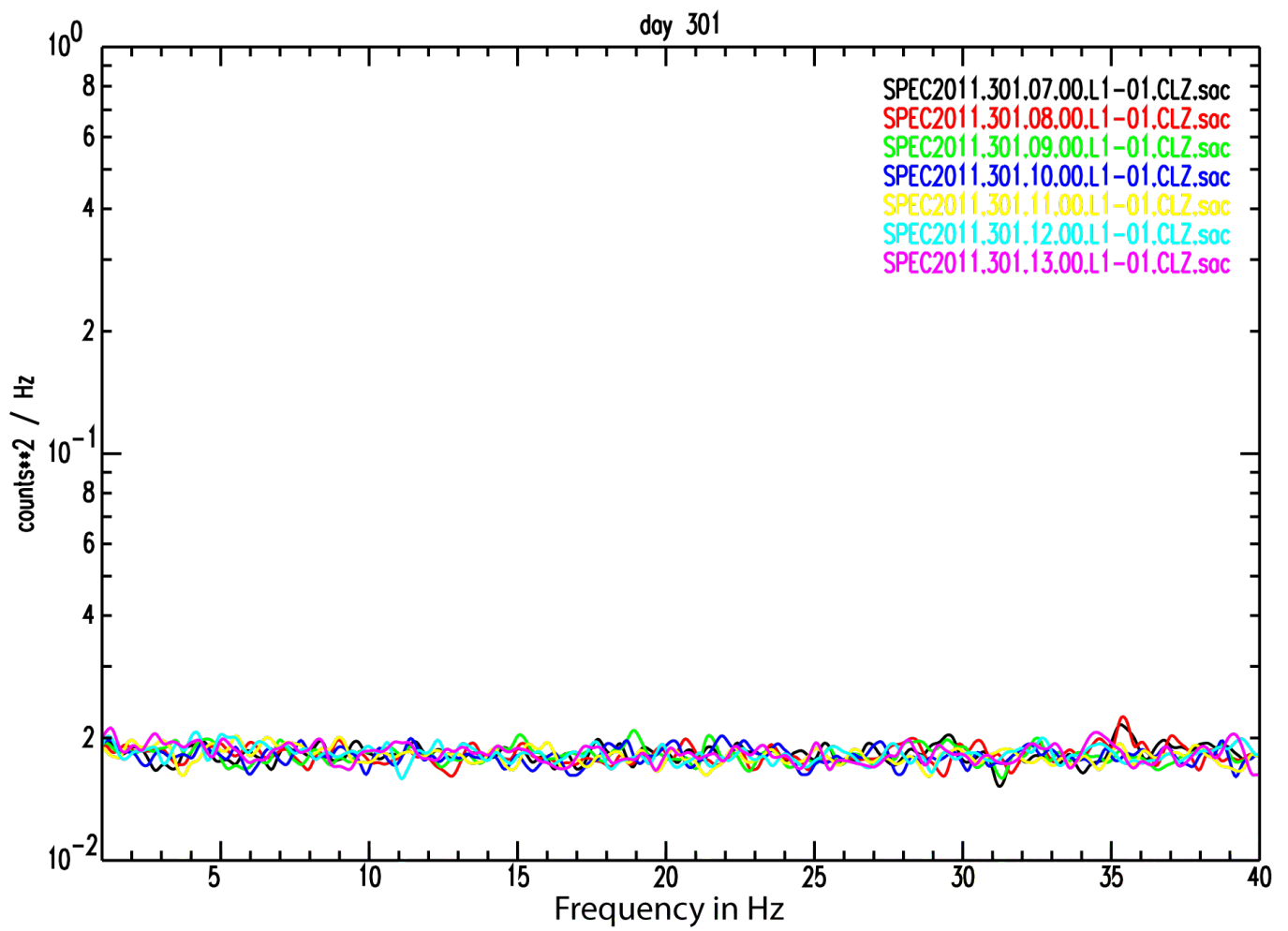

Figure 8. Power spectral density for each of seven consecutive hours after midnight local time (0700 through 1300 UT) on day 301 for the station 100 m from GZ along line 1 (refer to Fig 1). 


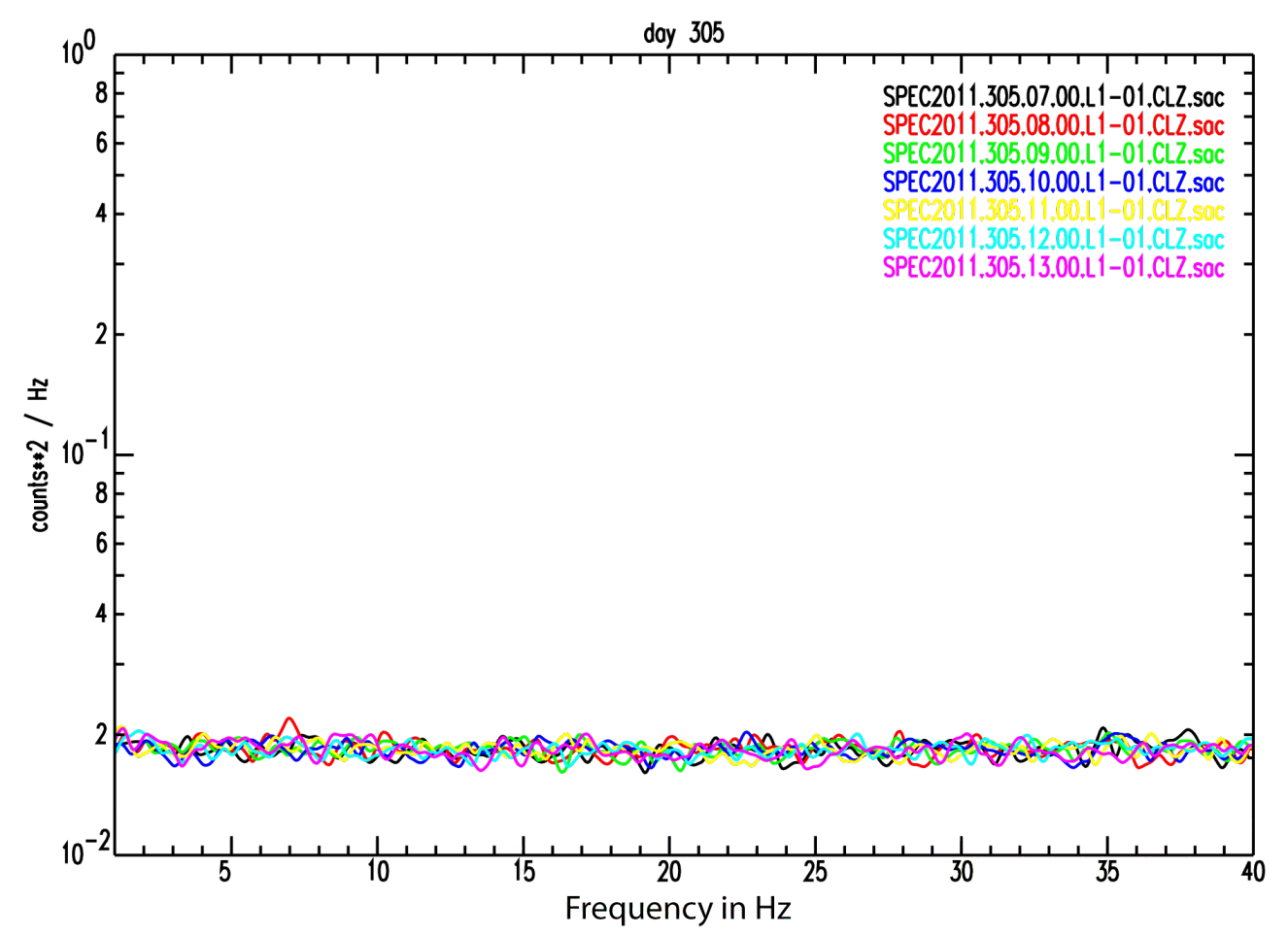

Figure 9. Power spectral density for each of seven consecutive hours after midnight local time (0700 through 1300 UT) on day 305 for the station 100 m from GZ along line 1 (refer to Fig 1).

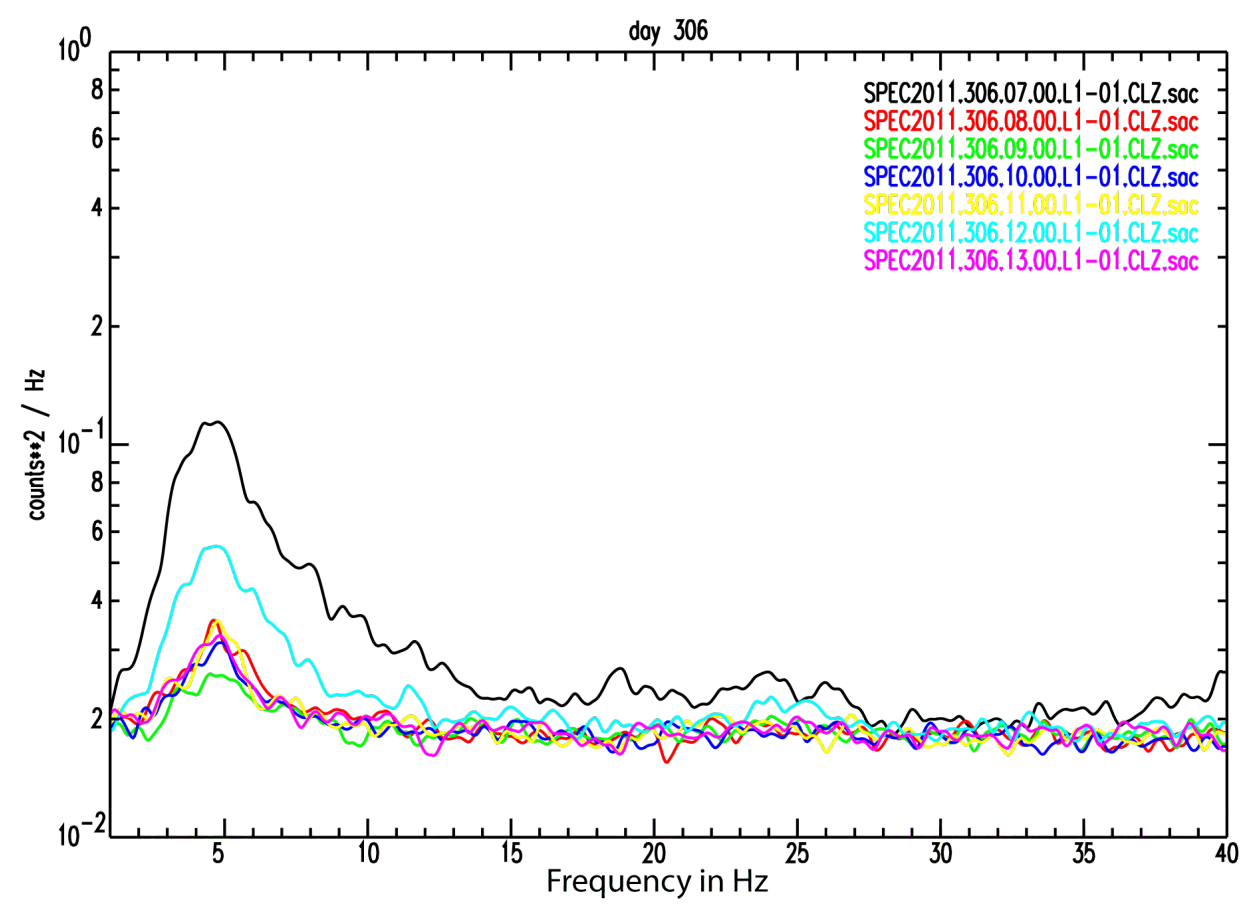

Figure 10. Power spectral density for each of seven consecutive hours after midnight local time (0700 through 1300 UT) on day 306 for the station 100 m from GZ along line 1 (refer to Fig 1). 


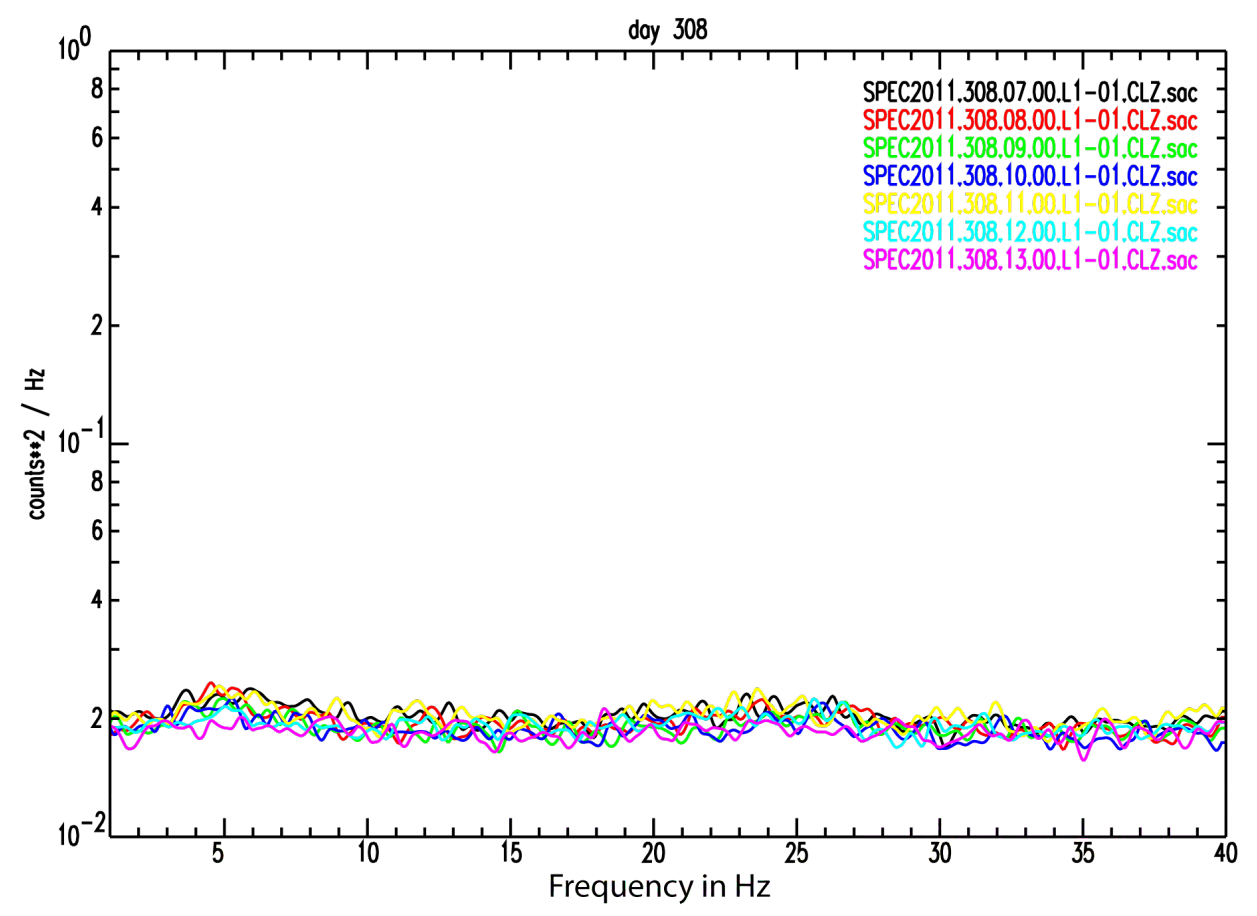

Figure 11. Power spectral density for each of seven consecutive hours after midnight local time ( 0700 through 1300 UT) on day 308 for the station $100 \mathrm{~m}$ from GZ along line 1 (refer to Fig 1).

The increased background noise levels at lower frequencies on days 299 and 300, just after the event, could indicate seismic activity near GZ resulting from the explosion although distinct events can not be detected. Such activity would be very local and should not be observed on more distant stations. Figs. 12-14 show the same quiet times on line L1 for day 300 at the 500 meter, 900 meter, and 1900 meter stations respectively: 


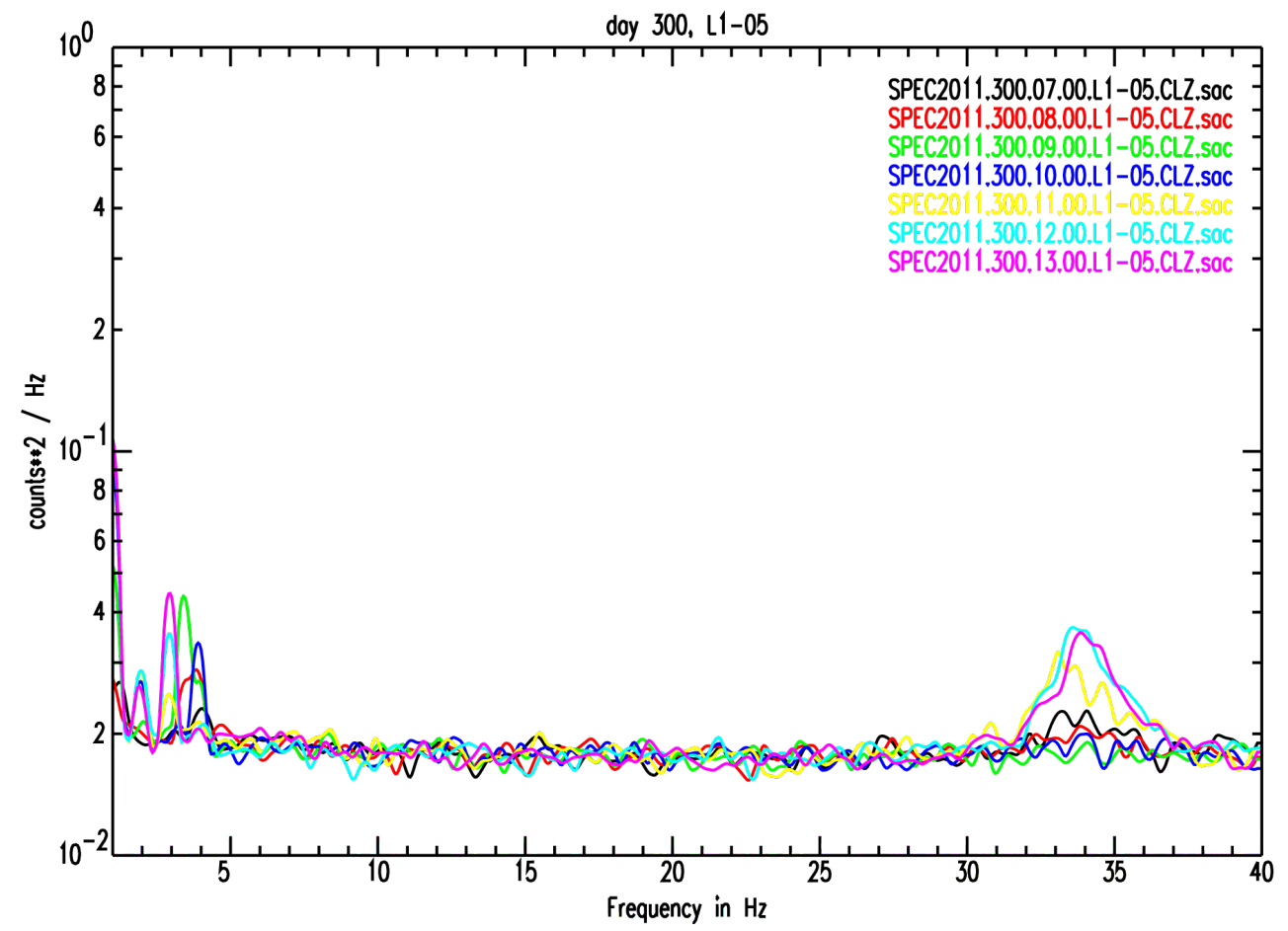

Figure 12. Power spectral density for each of seven consecutive hours after midnight local time (0700 through 1300 UT) on day 300 for the station $500 \mathrm{~m}$ from GZ along line 1 (refer to Fig 1 ).

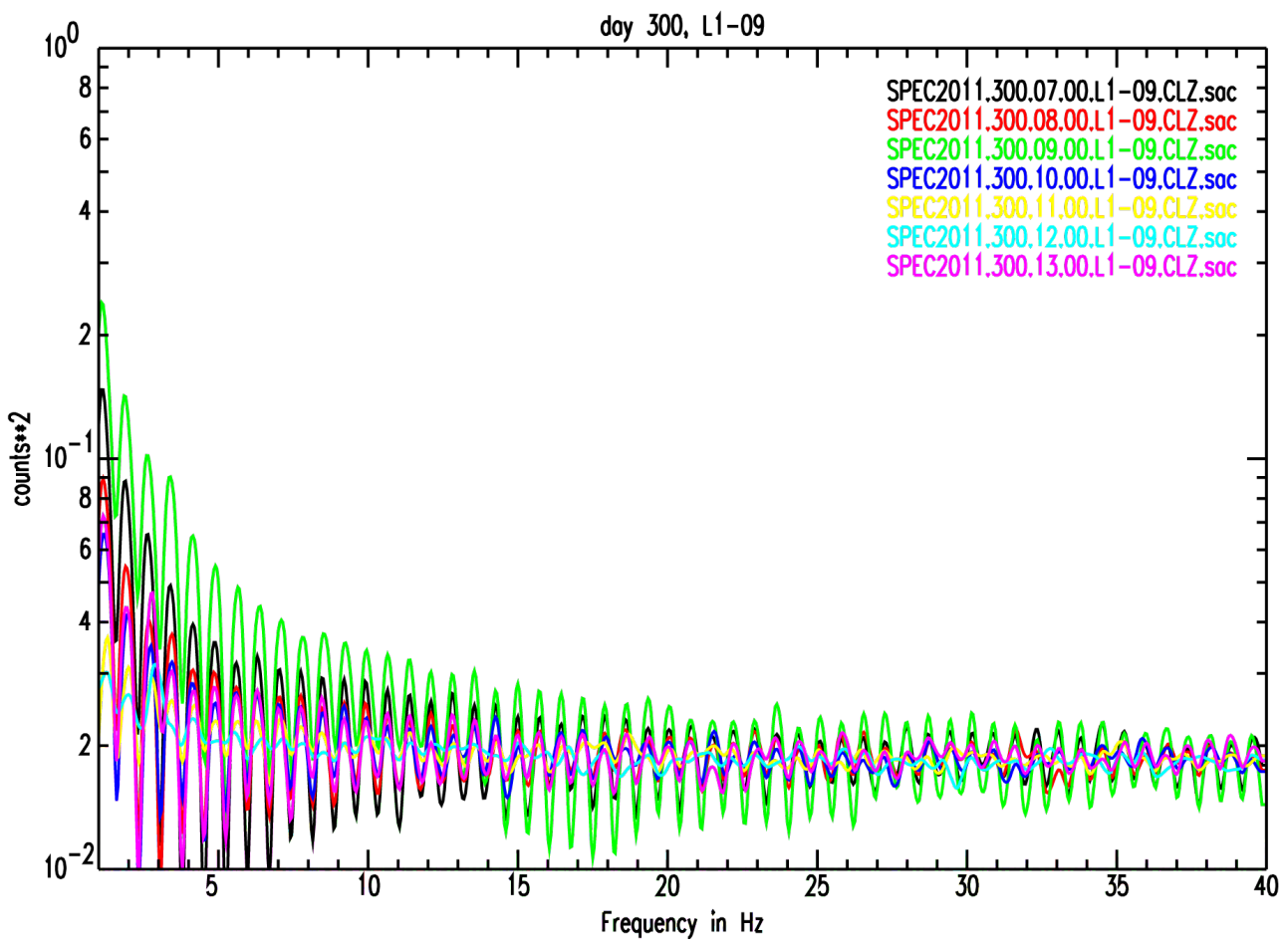

Figure 13. Power spectral density for each of seven consecutive hours after midnight local time (0700 through $1300 \mathrm{UT}$ ) on day 300 for the station $900 \mathrm{~m}$ from GZ along line 1 (refer to Fig 1). (Note that the scalloping of the spectra in this case may indicate that the signal is so low that what is calculated is digitization noise.) 


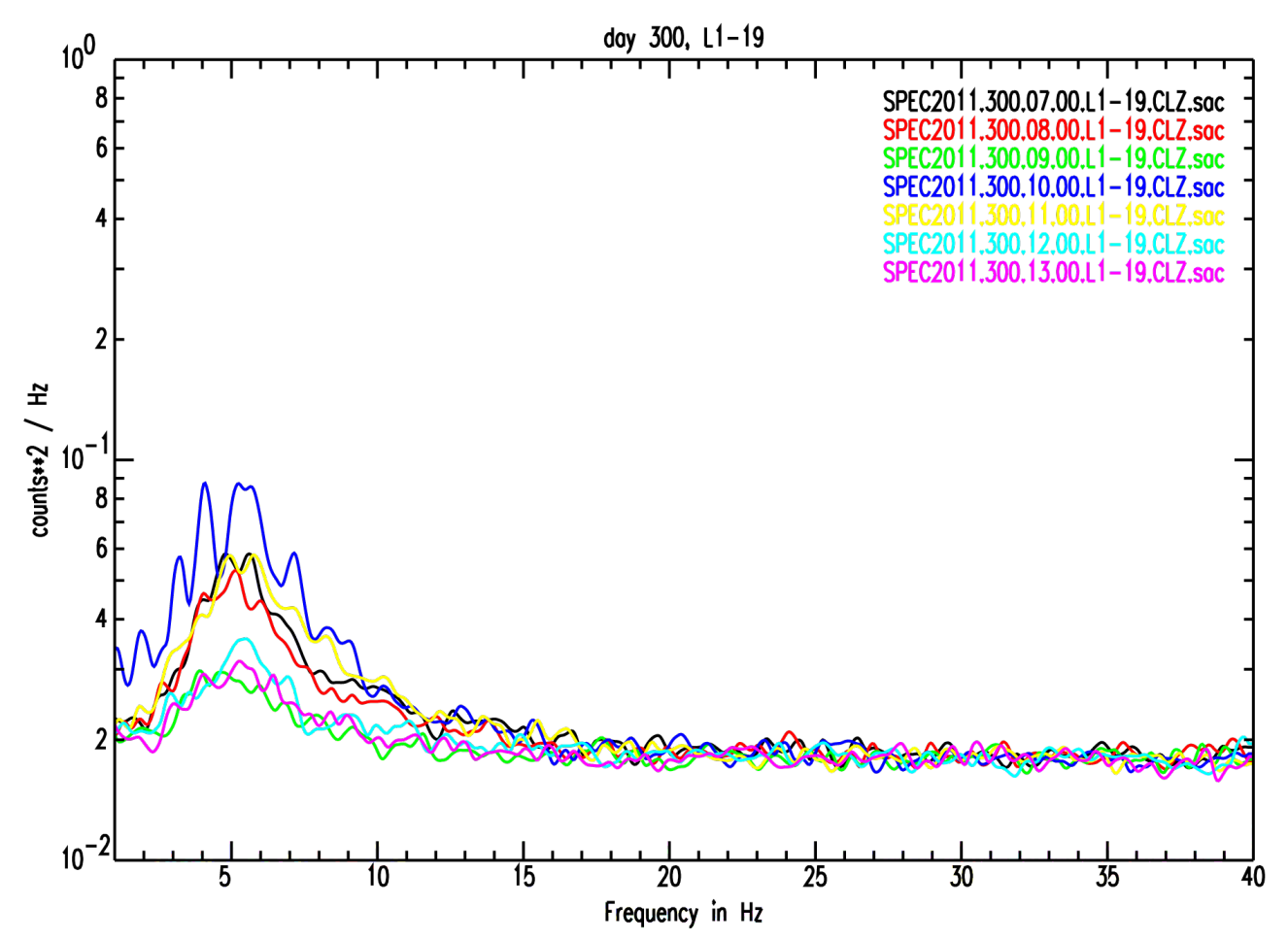

Figure 14. Power spectral density for each of seven consecutive hours after midnight local time (0700 through 1300 UT) on day 300 for the station $1900 \mathrm{~m}$ from GZ along line 1 (refer to Fig 1).

From the above spectra, it appears that the background noise is elevated at more distant stations (in particular, L1-19) from SPE2, making it unlikely that the source of the noise increase during hours 7 and 8 on day 300 is at or near the SPE2 explosion GZ.

For an additional look for a spatial signature of elevated noise, we plot power spectra from the first ring of stations (station 01 from each of lines L1 through L5) for the first four hours immediately after the SPE2 detonation (hours 20 through 23). Each power density spectrum is computed from one hour of data at each station. The spectra plotted in this way are compared, as successive hours of data, in Fig. 15. 

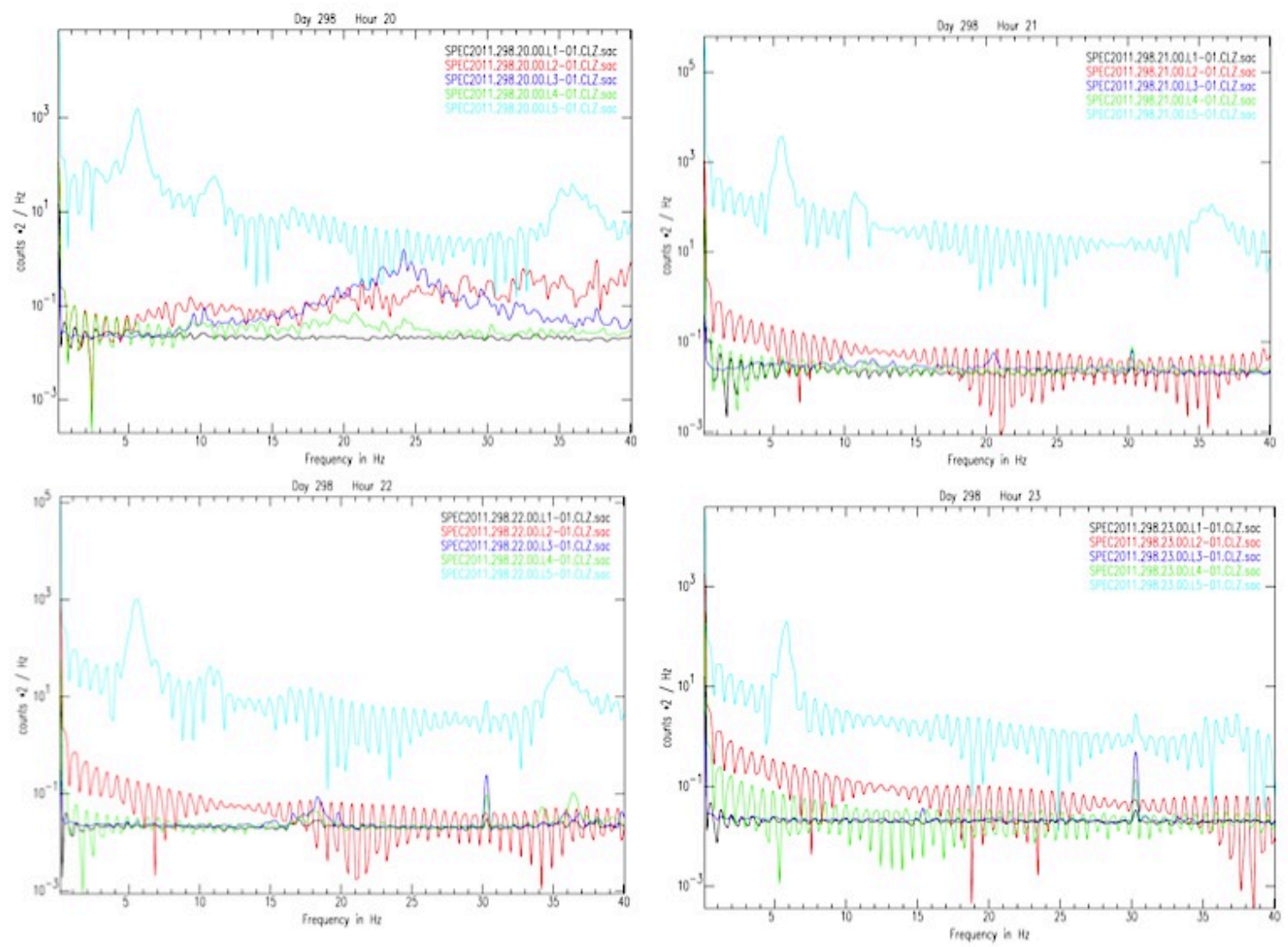

Figure 15. Power spectral density plots of one hour of data for all the stations in ring one (located $100 \mathrm{~m}$ from GZ on lines L1-L5) for hour 20 through 23 after detonation of SPE2 (detonation time hour 19).

There seems to be no elevated noise in the innermost ring around GZ during the first 4 hours following the explosion. Many of the spectra in Fig. 15 show scalloping that is probably due to digitization noise, and thus these spectra are not useful for the purposes of our noise analysis. We examined similar spectra for later periods after detonation with similar results.

\section{Discussion}

Aftershocks from SPE2, which had a yield 10 times larger than SPE1, were expected to occur. None were observed. The complete lack of observed aftershocks after both SPE1 and SPE2 is surprising, given the predictions of the aftershock model and given the very high detectability the network has in detecting aftershocks in the immediate vicinity of the SPE borehole.

Two processes may be at work to limit the aftershocks we can expect. The first is that, although both shots were over-buried from a scaled depth of burial viewpoint, they were still conducted at very shallow depths in an absolute sense. At such shallow depths (50 meters) significant levels of stress in the earth cannot be expected and consequently there may be little local residual stress to relieve. 
The second process that may be at work here is the recent history (geologically speaking) at the immediate site region. Two nuclear explosion tests were conducted within several kilometers of SPE GZ in the same granite stock that the SPE shots were in. The nuclear events were Hard Hat and Pile Driver (1962, and 1966 respectively). These explosions may have provided some stress relief within the granite stock well in advance of the SPE events.

We also examined borehole camera images of the borehole drilled back through the detonation point of SPE2 after the explosion. While there was evidence that fractures had been created and opened (as seen from wires caught in a fracture), there was no evidence of an open cavity created by the explosion. With no free face for movement of rock, there may be no opportunity for significant aftershocks.

We do not feel that the SPE1 and SPE2 results to date are sufficient to rule out the hypothesis that elevated seismic noise may persist around the vicinity of an explosion. These experiments were conducted using instruments available that were designed to measure a peak signal from an explosion, not to measure local noise levels. A set of data collections with dedicated experiments, using sensors and systems specific to noise measurement, should be tried to confirm the noise measurement hypothesis. Experiments using the seismic aftershock measurement systems (SAMS) currently owned and operated by the CTBT Organization would be ideal for this purpose.

\section{Acknowledgement}

The authors thank the National Nuclear Security Administration, Defense Nuclear Nonproliferation Research and Development (DNN R\&D) for their sponsorship of the National Center for Nuclear Security (NCNS) and its Source Physics Experiment (SPE) working group. This work was sponsored by the NNSA under award number DE-AC5206NA25946.

\section{References}

Adushkin, V. and A. Spivak, 1995, Aftershock of underground nuclear explosion, in Earthquakes Induced by Underground Nuclear Explosions: Environmental and Ecological Problems, NATO ASI Series: Series 2, Environment, R. Console and A. Nikolaev (Editors), 4, 35-49.

Ford, S., P. Labak, G. Leonard, A. Smith, and J. Sweeney, 2011, The use of explosion aftershock probabilities for on-site inspection planning, deployment, and reporting, (Abstract) Science and Technology 2011 Conference, Comprehensive Nuclear Test Ban Treaty Organization, June 8-10, 2011, Vienna, Austria.

Ford, S. R., and W. R. Walter (2010) Aftershock characteristics as a means of discriminating explosions from earthquakes, Bull. Seis. Soc. Amer., 100 
(1) 364-376, doi: 10.1785/0120080349.

Mellors, R. J., P. Harben, S. Ford, W. R. Walter, T. Hauk, S. Ruppert, A. Pitarka, and J. P. Lewis, 2012, SPE2 Far-field Seismic Data Quicklook, Lawrence Livermore National Laboratory report LLNL-TR-529834, 25 pp.

Rodgers, P.W., Frequency Limits For Seismometers As Determined From Signal-ToNoise Ratios. Part 1. The Electromagnetic Seismometer, BSSA, Vol. 82, No. 2, pp 10711098, Apr, 1992.

Ryall, A. and W. U. Savage, 1969, A comparisonof seismological effects for the Nevada underground test BOXCAR with natural earthquakes in the Nevada region, J. Geophys. Res., 24, 4281-4289.

Sweeney, J. and P. Harben, 2011, Aftershock analysis for SPE1, Lawrence Livermore National Laboratory report LLNL-TR-512872, 13 pp.

\section{MATLAB Script for Contouring}

Below is a listing of the script used to create the plots from a 3 column Excel file input:

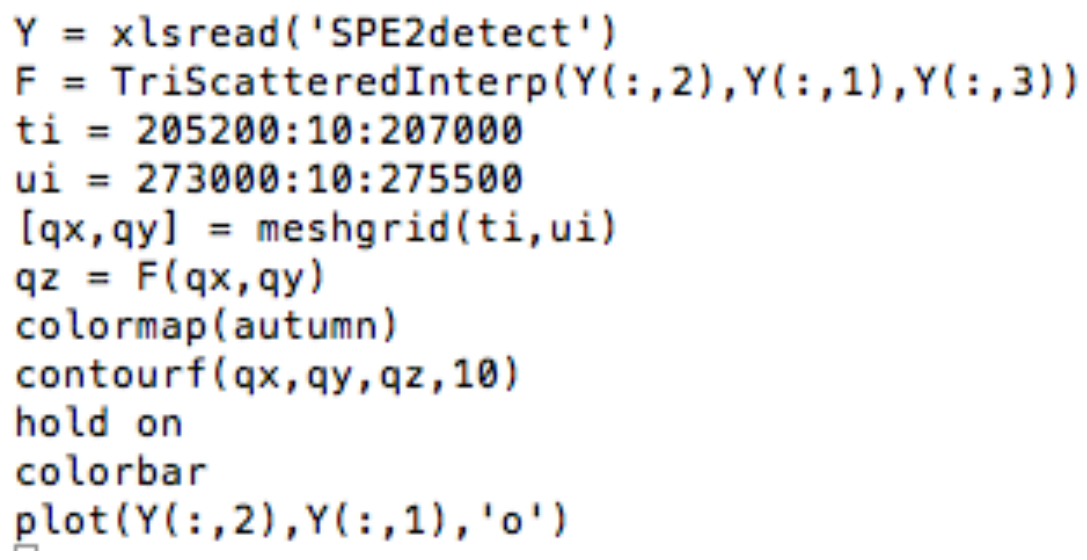

\section{SAC Script for Computing Power Density}

(for a 60 minute file, sampled at $500 \mathrm{~Hz}, 180,000$ points in the file)

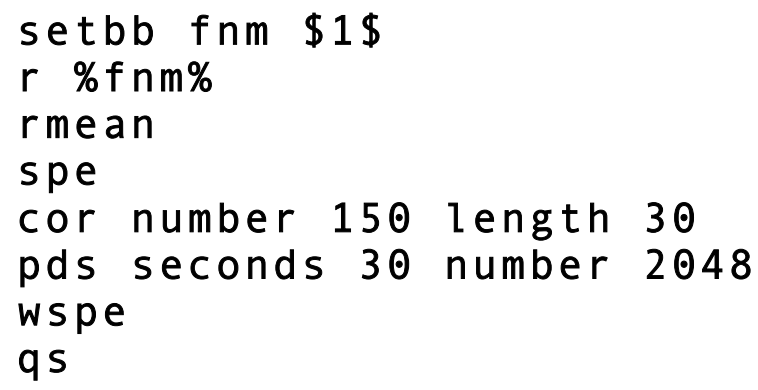


$r$ spe

interpolate begin .1 delta .05 npst 800

w SPEC\%fnm\% 